\title{
In search of stable visible light absorbing photocatalysts: gold nanoclusters $^{\S}$
}

\author{
BARNALI MONDAL, MOHAN RAJ ANTHONY RAJ and V RAMAMURTHY* (D \\ Department of Chemistry, University of Miami, Coral Cables, Miami, USA \\ E-mail: murthy1@miami.edu
}

MS received 14 August 2018; revised 31 August 2018; accepted 3 September 2018; published online 26 September 2018

\begin{abstract}
Photochemical reactions are beginning to play an important role in the synthesis of complex organic molecules. The impetus to employ light initiated photoreactions as a synthetic tool derives from the fact that these reactions require no reagents except light. The abundance of sunlight has prompted the chemists to search for the visible light absorbing sensitizers (catalysts) to initiate the desired reactions. Our goal in this context is to develop stable and readily available catalysts that would function under sunlight. In this manuscript, we present the results of our experiments with gold nanoclusters (AuNCs) as a visible light absorbing catalysts. AuNCs absorb and emit in the visible region, soluble in water and transfers electron to suitable acceptors. Employing a series of acceptors we found that excited AuNC can transfer one electron to any acceptor whose reduction potential is above $-1.1 \mathrm{eV}$. In the excited state, AuNC does not accept electrons. Also, it did not serve as an energy transfer sensitizer even with molecular oxygen. We are optimistic that AuNP and AuNC could be developed into a stable and water-soluble visible light absorbing photocatalysts to perform useful photoreactions.
\end{abstract}

Keywords. Nanoclusters; photocatalysis; electron transfer; emission quenching; visible light photocatalysis.

\section{Introduction}

Prompted by the need to carry out organic synthesis under 'green' and 'sustainable' conditions interest in visible light photocatalysis (VLPC) has grown during the last decade. ${ }^{1-3}$ Energy and electron transfer concepts developed by pioneers during 195080 have been valuable for the development of this topic. ${ }^{4}$ Among the VLPC enthusiasts, the term "sensitizer' used in photochemical literature has taken a new label namely, 'catalyst'. Although numerous visible light absorbing organic dyes that could be used as catalysts (sensitizers) are available, their poor stability and tendency to aggregate have forced chemists to look for $\mathrm{Ru}, \mathrm{Re}, \mathrm{Rb}$ complexes and metal aggregates that absorb in the visible region as photocatalysts. In this context, two types of metal nano-aggregates with different properties have attracted attention. One is metal nanoparticles (NPs) $)^{5-11}$ and the other is metal nanoclusters (NCs). ${ }^{12-18}$ For example, metal nanoparticles of gold, silver and platinum are characterized by their distinct surface plasmon band in the visible spectrum extending to red while gold nanoclusters have a broad absorption in the visible region. ${ }^{19}$ While metal nanoparticles are non-emissive, nanoclusters emit in the visible spectrum. ${ }^{20-22}$ The nanoclusters are usually smaller in size than nanoparticles and their absorption and emission depend on their size and the stabilizing ligand. The properties of metal nanoclusters, in addition, depend on the method of preparation. The recent interest in this ultra-small nanoclusters is due to their molecular like properties and emissive nature. The emissive property of these Au-thiolate nanoclusters, their low toxicity, ultra-small size, and good biocompatibility, make them ideal as bioimaging probes.

\footnotetext{
*For correspondence

$\S$ Dedicated to Professor M V George on the occasion of his $90^{\text {th }}$

Birth Anniversary.
}

Electronic supplementary material: The online version of this article (https://doi.org/10.1007/s12039-018-1553-x) contains supplementary material, which is available to authorized users. 
A recent report of an easy and reliable procedure to synthesize thiolate-protected water-soluble gold nanoclusters of $1-3 \mathrm{~nm}$ diameter. ${ }^{23,24}$ prompted us to explore their use as a visible light absorbing photocatalysts. Our interest in these systems stems from their possible use as energy and electron transfer sensitizers to initiate organic phototransformations. ${ }^{25-29}$ With this in mind, we have examined the electron and energy transfer properties of AuNC by quenching their emission with various organic molecules and oxygen. Our ultimate goal is to identify metal nanoclusters and nanoparticles that could act as an energy or electron transfer catalysts (sensitizers) in water.

\section{Experimental}

All the experimental details that include synthesis and characterization of AuNC, procedures for recording emission and lifetime are provided as Supplementary Information.

\section{Results and Discussion}

Thiolate-stabilized AuNC are commonly synthesized by reducing $\mathrm{Au}(\mathrm{I})$ thiolate complexes using a reducing agent such as $\mathrm{NaBH}_{4}$. These clusters show emission in the blue to the near-IR region with low quantum yield. Recent research has established that slow decomposition of large Au-nanocrystals or Au-thiolate complex can also be used to synthesize AuNC. A facile onepot synthesis of Au-thiolate has been reported recently by Xie and co-workers (Scheme 1). For the current investigation, L-glutathione protected gold nanoclusters were synthesized using the method reported by Xie et al. ${ }^{23,24}$ By this procedure, as the solution aged for $24 \mathrm{~h}$, nanoclusters having a few-gold-atom core capped with $\mathrm{Au}(\mathrm{I})$ thiolate complex shell were formed. These AuNCs were characterized by their absorption, emission and ${ }^{1} \mathrm{H}$ NMR spectra, TGA and DLS data and TEM (Figures S1-S5 in Supplementary Information). These clusters absorb both in UV and a visible region extending up to $500 \mathrm{~nm}$. These AuNCs exhibited emission in the region $500-800 \mathrm{~nm}$ with a maximum at $612 \mathrm{~nm}$. The absorption and emission spectra of above synthesized
Au nanoclusters are shown in Figure 1. These spectra are consistent with the ones reported in the literature. It is reported that these clusters consist of a mixture of different sizes consisting of 29-43 Au atoms capped with glutathione molecules. The clusters prepared in this study we believe are a mixture and not a well-defined one with a specific number of Au atoms. Having synthesized small AuNCs we were interested to examine whether these would act as electron and energy transfer sensitizers. Other workers have employed such types of clusters in the context of solar energy storage and water splitting. ${ }^{30,31}$ However, to our knowledge, these have not been used as sensitizers in organic photochemistry. The emissive nature distinguishes these clusters from larger nanoparticles that have characteristic plasmonic properties and do not emit.

With our interest to examine whether these clusters could act as good electron transfer agents, we conducted fluorescence quenching experiments with various organic electron acceptors and donors. The quenchers examined and their redox potential data are listed in Scheme 2. Electron transfer properties of these clusters were probed by recording fluorescence and measuring the excited state lifetime in presence of quenchers. Cluster emission was quenched by methyl viologen (2) and its derivative (1). Stern-Volmer plots of quenching by $\mathbf{1}$ and $\mathbf{2}$ shown in Figures 2 and 3 indicate that the $\mathrm{I}_{0} / \mathrm{I}$ and $\tau_{0} / \tau$ do not overlap. Since there

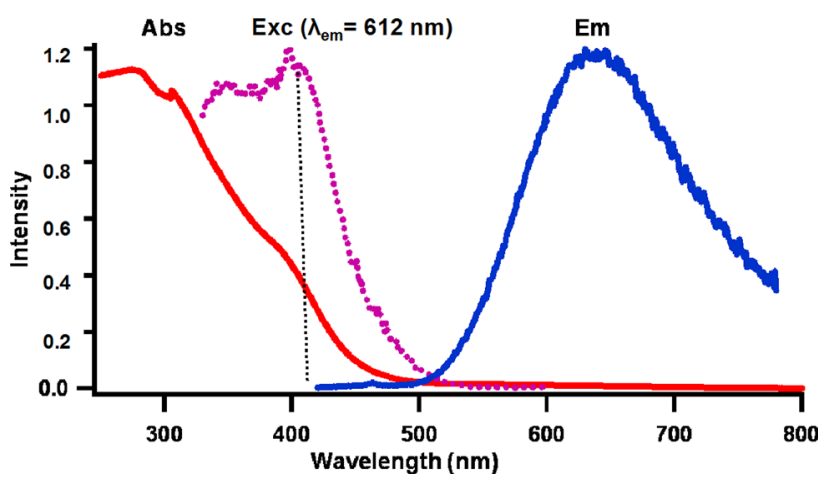

Figure 1. Normalized absorbance (red line), excitation (pink line) monitored at $612 \mathrm{~nm}$ and emission spectra (blue line, $\lambda_{\mathrm{ex}}=400 \mathrm{~nm}$ ) of AuNC.<smiles>N[C@@H](CCC(=O)N[C@@H](CS)C(=O)NCC(=O)O)C(=O)O</smiles>

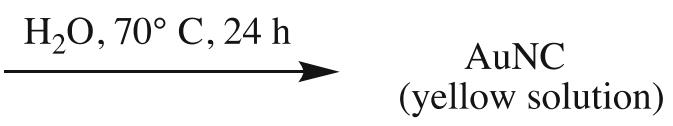

L-Glutathione

Scheme 1. Method used to synthesize Au-glutathione nanocluster (AuNC). 


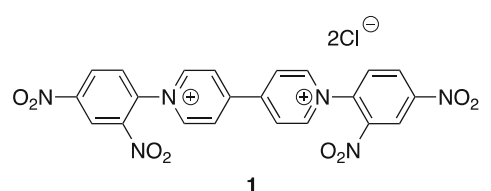

$(-0.40 \mathrm{~V})$

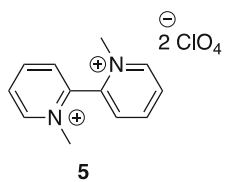

$(-0.73 \mathrm{~V})$

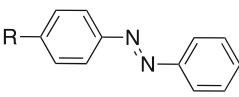

$R=$ methyl (13a)

$\mathrm{R}=$ ethyl $\quad(14 \mathrm{a}) \quad \approx(-1.36 \mathrm{~V})$

$\mathrm{R}=$ propyl $\quad$ (15a)
$\mathrm{O}_{2}$

$(-0.78 \mathrm{~V})$

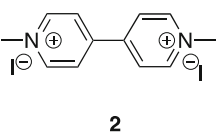

$(-0.45 \mathrm{~V})$

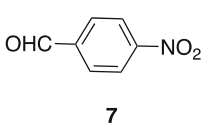

$(-0.86 \mathrm{~V})$

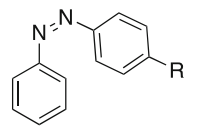

$\mathrm{R}=$ methyl (13b)

$\mathrm{R}=$ ethyl $\quad(14 \mathrm{~b}) \quad \approx(-1.40 \mathrm{~V})$

$R=$ propyl $\quad(15 b)$

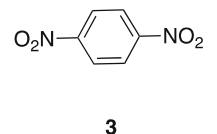

$(-0.69 \mathrm{~V})$

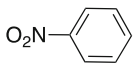

8

$(-1.08 \mathrm{~V})$<smiles>COc1ccc(OC)cc1</smiles>

9

(1.34 V)<smiles>Nc1cc(N)c(N)cc1N</smiles>

4

(-0.71V)

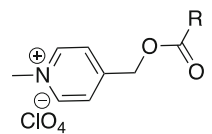

$\mathrm{R}=$ phenyl (11)

$\mathrm{R}=$ benzy $\mid$ (12)

Scheme 2. Structures of molecules used as acceptors and donors. Redox potentials reported are with respect to SCE in $\mathrm{CH}_{3} \mathrm{CN}$ except in $\mathbf{1}$ where it is with respect to SCE in DMF. Reduction potentials of $\mathbf{1 - 8}$ and 11-15 and oxidation potentials of $\mathbf{9}$ and $\mathbf{1 0}$ are provided in parenthesis.

(a)

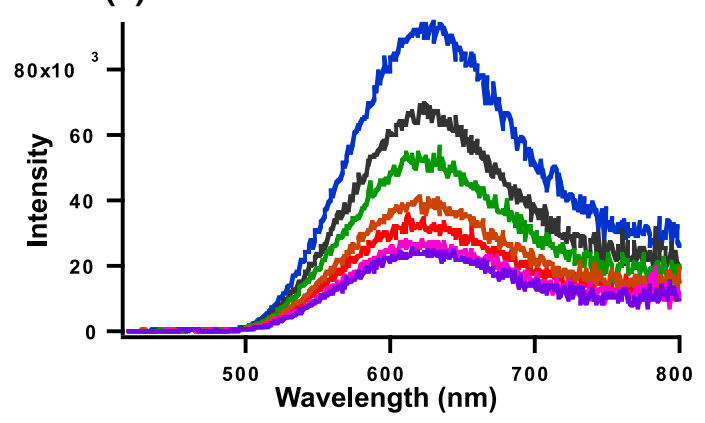

(b)

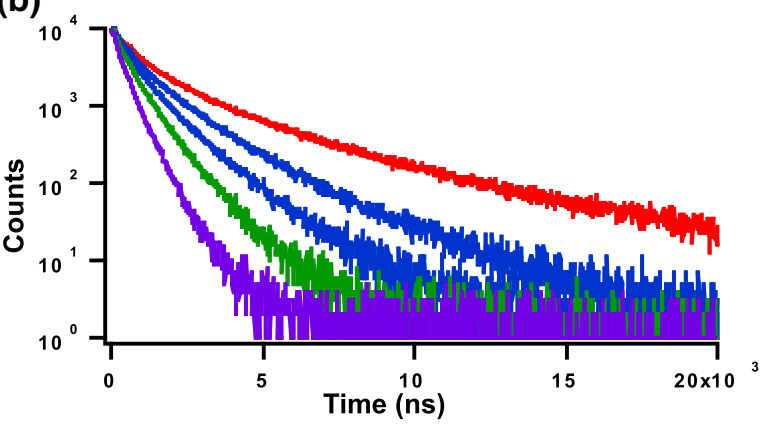

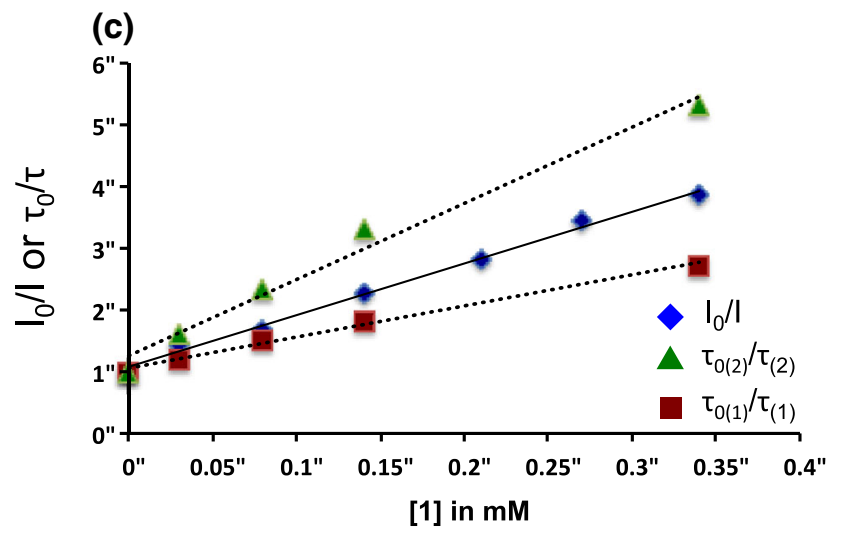

Figure 2. Quenching of emission of AuNC by 1 in $\mathrm{H}_{2} \mathrm{O}$. (a) Emission spectra; (b) Lifetime measurements; (c) Stern-Volmer plots for emission and lifetime. $\lambda_{\mathrm{ex}}=400 \mathrm{~nm}, \lambda_{e m}=600 \mathrm{~nm},[\mathrm{AuNC}]=10^{-4} \mathrm{M}$; $[1]=$ 0 to $3.4 \times 10^{-3} \mathrm{M}$. The excited AuNC decayed via two exponentials and both are plotted in (c). 
(a)
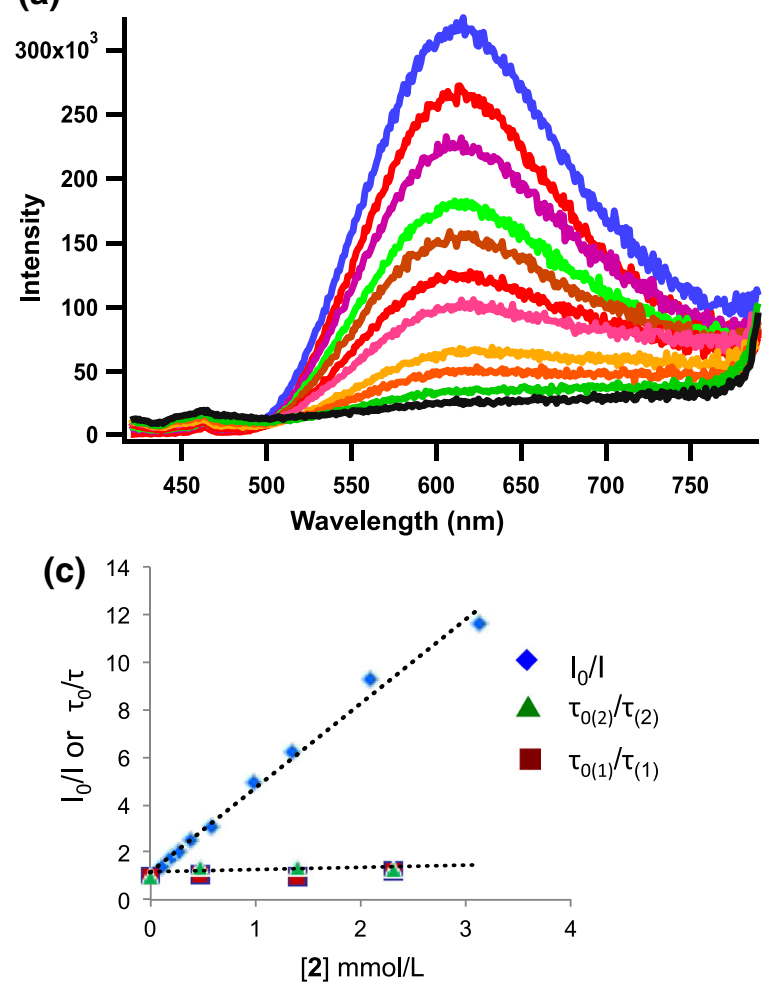

(b)

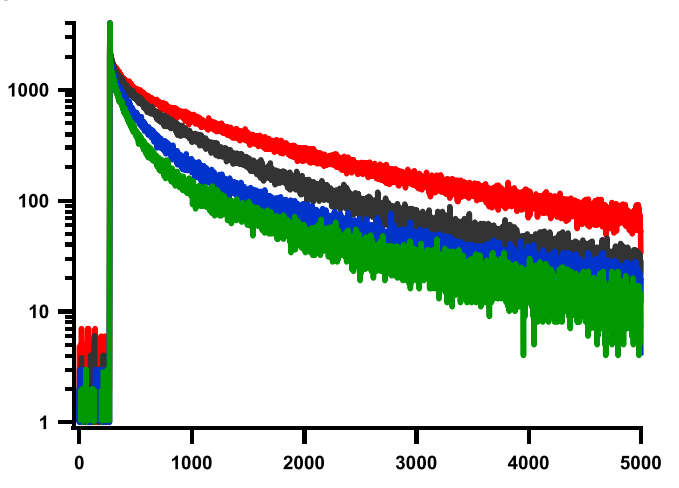

(d) 2.5

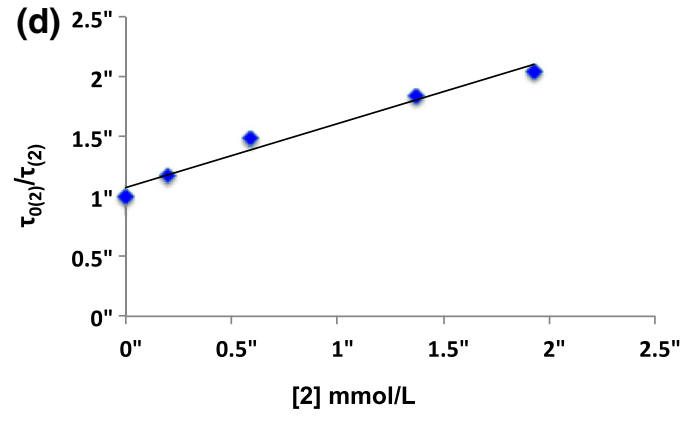

Figure 3. Quenching of emission of AuNC by 2 in $\mathrm{H}_{2} \mathrm{O}$. (a) Emission spectra; (b) Lifetime measurements; (c) Stern-Volmer plot for emission and lifetime. $\lambda_{\mathrm{ex}}=400 \mathrm{~nm}, \lambda_{\mathrm{em}}=600 \mathrm{~nm},[\mathrm{AuNC}]=10^{-4} \mathrm{M}$; [2] $=0$ to $3.1 \times 10^{-3} \mathrm{M}$. The AuNC decayed via two exponentials and both are plotted in (c). Figure (d) provides a Stern-Volmer plot for the faster component of the decay.

are changes in lifetimes, the quenching is not completed by a static process. If this is truly a static process the donor (AuNC) and the acceptor (viologens) should be in physical contact prior to excitation. We were curious to know whether the viologen that is included within a cucurbiturils (CB) would be able to establish contact and quench the AuNC emission. As illustrated in Figure 4, CB[7] included 2 (i.e., 2@CB[7]) failed to quench the cluster emission. This suggested that AuNC and the electron acceptor have to be in physical contact for electron transfer to occur. Whether it is static or dynamic the electron transfer is prohibited by the walls of the host CB[7]. Due to lack of time-resolved transient absorption facilities, we did not measure the rate of electron transfer. However, the recent report by Kamat's group indicated that AuNC is quenched by $\mathbf{2}$ at $>5 \times 10^{10} \mathrm{M}^{-1} \mathrm{~s}^{-1}$ that is higher than the rate of diffusion. ${ }^{28}$ This is consistent with our conclusion of the quenching proceeding by a static process. Since the excited state energies of $\mathbf{1}$ and $\mathbf{2}$ are expected to be above that of AuNC we believe that the process by which the quenching occurs is via electron transfer.

To probe whether AuNC would also act as an energy transfer sensitizer, we chose oxygen as the acceptor.

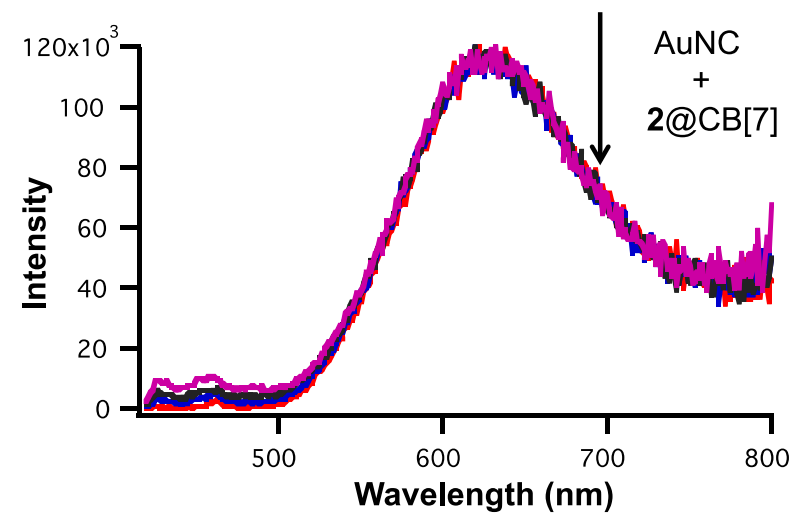

Figure 4. Emission spectra of AuNC upon addition on 2@CB[7] (CB[7] methyl viologen complex). [AuNC] $=10^{-4}$ $\mathrm{M} ;[2 @ \mathrm{CB}[7]]=0$ to $2 \times 10^{-4} \mathrm{M}$.

We believed that oxygen with excited singlet energy of $22 \mathrm{kcal} / \mathrm{mol}$ would be an ideal acceptor for energy transfer from excited AuNC. As illustrated in Figure 5 molecular oxygen quenched the emission of AuNC. Quenching of AuNC emission by $\mathrm{O}_{2}$ was studied in three different solvents, $\mathrm{H}_{2} \mathrm{O}$, dimethyl sulfoxide and methanol. Since the solubility of oxygen differed in 

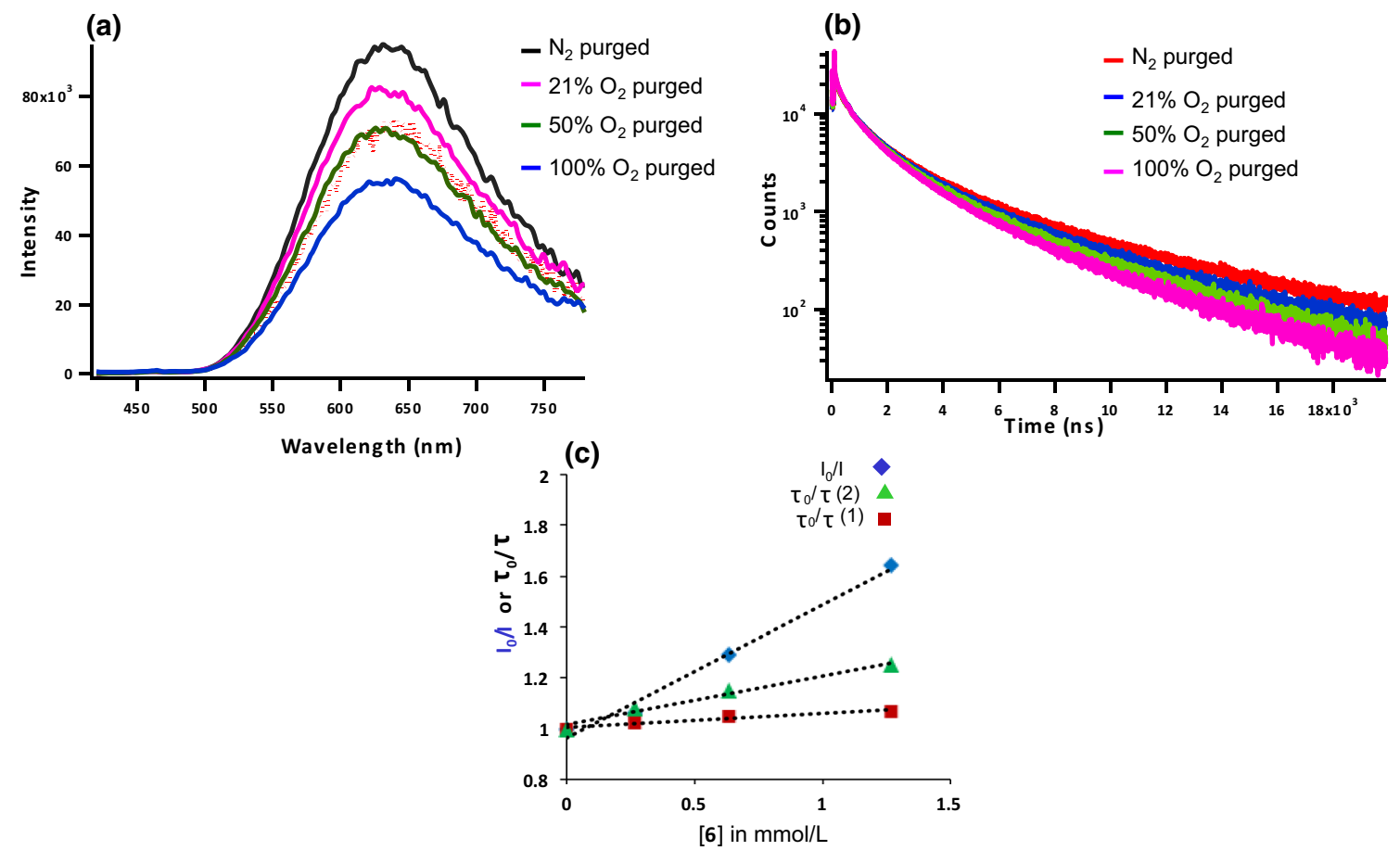

Figure 5. Quenching of emission of AuNC by oxygen in $\mathrm{H}_{2} \mathrm{O}$. (a) Emission spectra; (b) Lifetime measurements; (c) Stern-Volmer plot for emission and lifetime. $\lambda_{\mathrm{ex}}=400 \mathrm{~nm}, \lambda_{\mathrm{em}}=600 \mathrm{~nm}$, [AuNC] $=10^{-4}$ M. Solution was purged with oxygen-nitrogen mixture; the percentage of oxygen is indicated above. The AuNC decayed via two exponentials and both are plotted in (c).

(a)

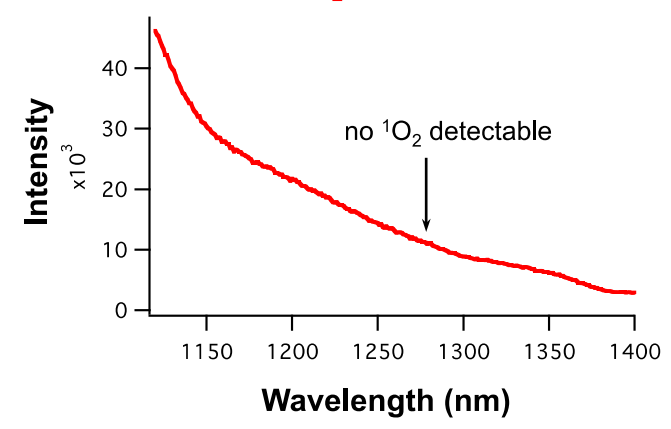

(b)

$\left[\mathrm{O}_{2}\right]=2.2 \mathrm{mM}$

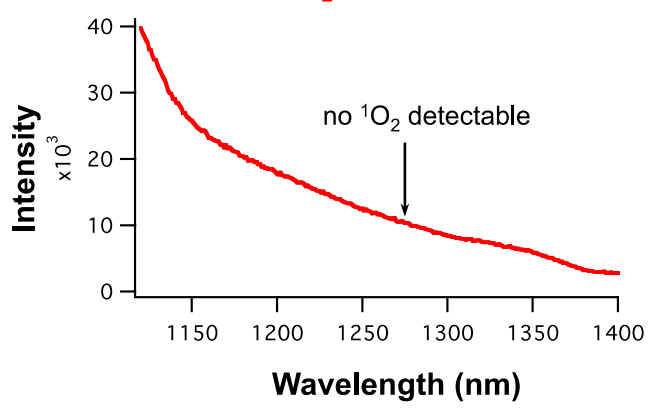

Figure 6. Emission spectra of AuNC solution saturated with oxygen. (a) $\mathrm{D}_{2} \mathrm{O}$ and (b) DMSO-d 6 . $\lambda_{\text {ex }}: 400 \mathrm{~nm}$.

these solvents, we expected the rate of quenching would vary. In methanol where the $\mathrm{O}_{2}$ dissolves better than in the other two solvents, the cluster emission was quenched more efficiently than in $\mathrm{H}_{2} \mathrm{O}$ and DMSO (Figures S11-S12 in Supplementary Information). If the quenching is due to energy transfer singlet oxygen should be generated in solution. Singlet oxygen in principle can occur from either excited singlet (when the $\mathrm{S}_{1}-\mathrm{T}_{1}$ gap is more than $22 \mathrm{kcal} / \mathrm{mol}$ ) or from triplet when it is above $22 \mathrm{kcal} / \mathrm{mol}$. However characteristic singlet oxygen emission (1268 nm) was not detected when an oxygen saturated solution of AuNC was excited at $400 \mathrm{~nm}$ (Figure 6). This suggested that energy transfer is not the process by which oxygen quenches the AuNC emission. However, the generation of singlet oxygen from well-defined $\mathrm{Au}_{25}(\mathrm{SR})_{18}$ cluster has been reported in the literature. ${ }^{32}$ The fact that the emission of AuNC is quenched by oxygen suggested that most likely it occurs by electron transfer. Our attempts to detect the product of electron transfer namely superoxide anion were not successful.

The ability of oxygen to quench AuNC emission suggested that it could oxidize systems with a reduction potential of at least $-0.78 \mathrm{eV}$. To determine the 


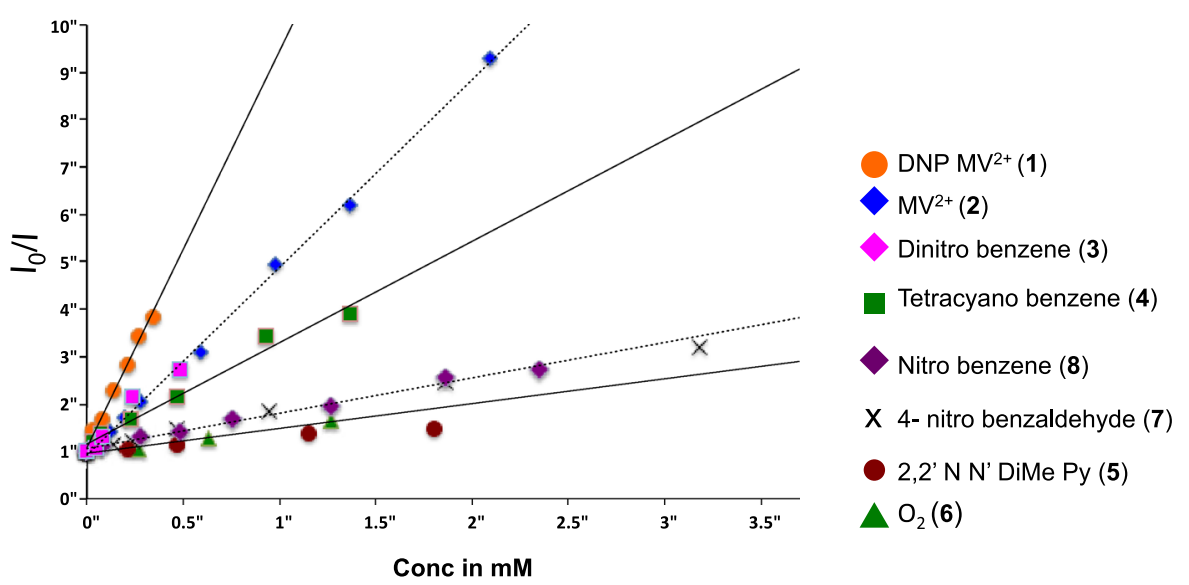

Figure 7. Stern-Volmer plots for fluorescence quenching experiments with various acceptors.

(a)

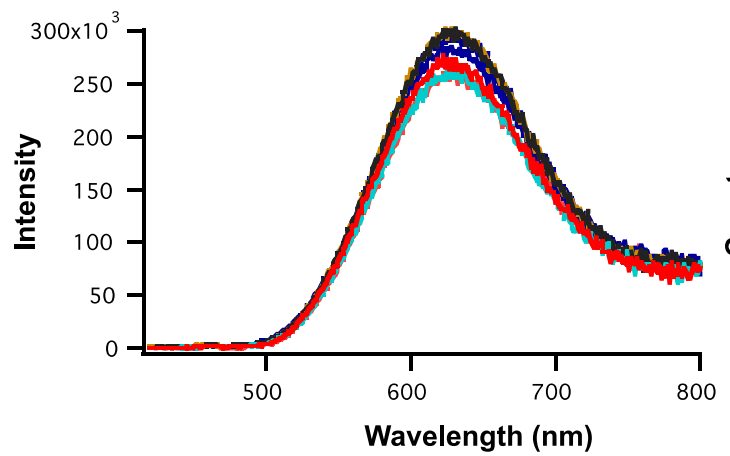

(b)

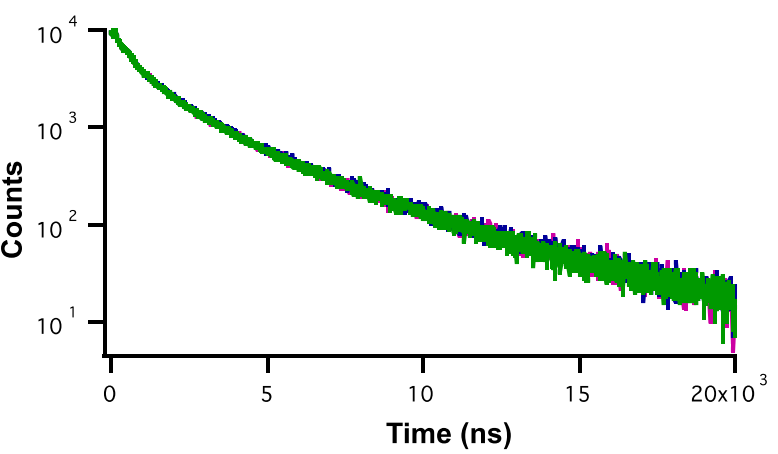

Figure 8. AuNC emission in presence of 1,3,5-trimethoxy benzene in $\mathrm{H}_{2} \mathrm{O}$. (a) Emission spectra; (b) Lifetime measurements. $\lambda_{\text {ex }}=400 \mathrm{~nm}, \lambda_{\mathrm{em}}=600 \mathrm{~nm},[\mathrm{Au} \mathrm{NCs}]=10^{-4} \mathrm{M},[\mathbf{1 0}]=0$ to $2.0 \times 10^{-3} \mathrm{M}$.

oxidation power of the AuNC we used several quenchers with reduction potentials varying between -0.40 and $-1.5 \mathrm{eV}$. The list of quenchers included 2,4-dinitro phenol (3), 1,2,4,5-tetracyano benzene (4), 2,2'-bipyridinium N, N' dimethyl (5), 4-nitro benzaldehyde (7), and nitro benzene $(\mathbf{8})$. Their structures and reduction potentials are provided in Scheme 2. Stern-Volmer plots for quenching by these acceptors are shown in Figure 7. Individual emission and lifetime quenching spectra and traces are provided in SI (Figures S6-S10, Supplementary Information). Although nitrobenzene quenched the emission, the pyridinium derivatives $\mathbf{1 1}$ and $\mathbf{1 2}$ did not. This suggested that the limitation of AuNC to reduce organic molecules resided somewhere in between $-1.0 \mathrm{eV}$ and $-1.5 \mathrm{eV}$. Based on the results shown in Figure 7, we believe that AuNC can reduce organic molecules whose potential is above $-1.1 \mathrm{eV}$. This most likely represents the limiting reduction ability of the nanoclusters investigated here. ${ }^{33-35}$ To probe whether AuNC has similar property we used di- and trimethoxy- benzenes as electron donors (Scheme 2). As illustrated in Figure 8 no quenching was observed even with a good electron donor such as 1,3,5-trimethoxy benzene.

Thus the results with the electron donors and acceptors listed in Scheme 2 lead us to conclude that the $\mathrm{AuNC}$ is an electron donor and not an electron acceptor. Also, results with oxygen suggested that AuNC is not an energy donor. As mentioned in the Introduction section, the desire to identify visible light absorbing light-stable sensitizers drew our attention to gold nanoparticles and clusters. In this context, we were particularly attracted by the studies of Falvey's group in which they demonstrated that carboxylic acids could be released by a photo-triggering reaction initiated by AuNP through an electron transfer process (Scheme 3, eq. 1). ${ }^{36}$ In their studies dithiothreitol served as the primary electron donor to excited AuNP. We experimented the same reaction with AuNC without an additional electron donor (Scheme 3, eq. 2). As shown in Figure 9, as expected based on reduction potential, AuNC emission was not significantly quenched by the photoprotected esters $\mathbf{1 1}$ 


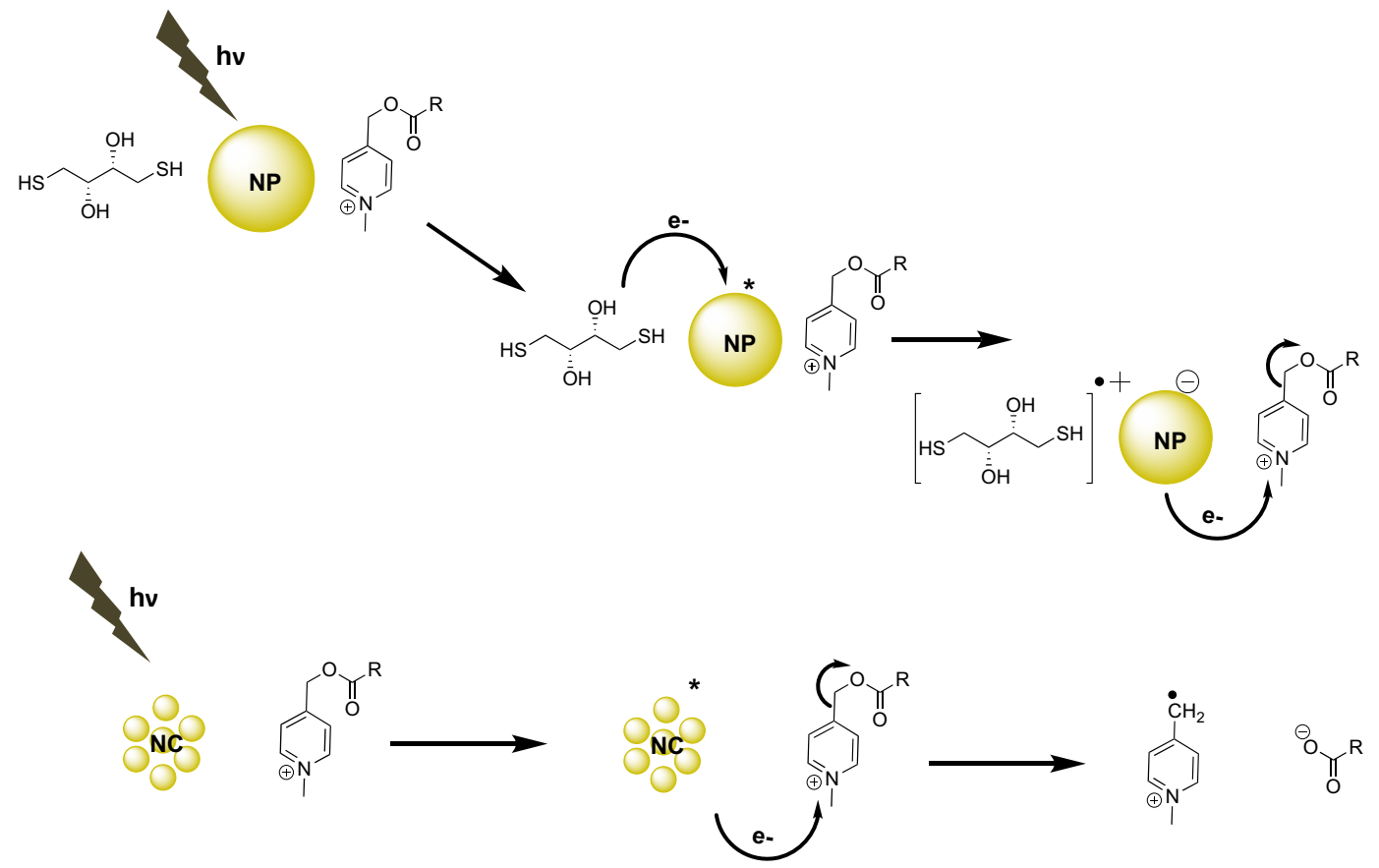

Scheme 3. (Top): Steps during decarboxylation of N-methylpicolinium ester triggered by visible light absorbing AuNP. (Below) Visualized steps during decarboxylation of N-methylpicolinium ester triggered by visible light absorbing AuNC.
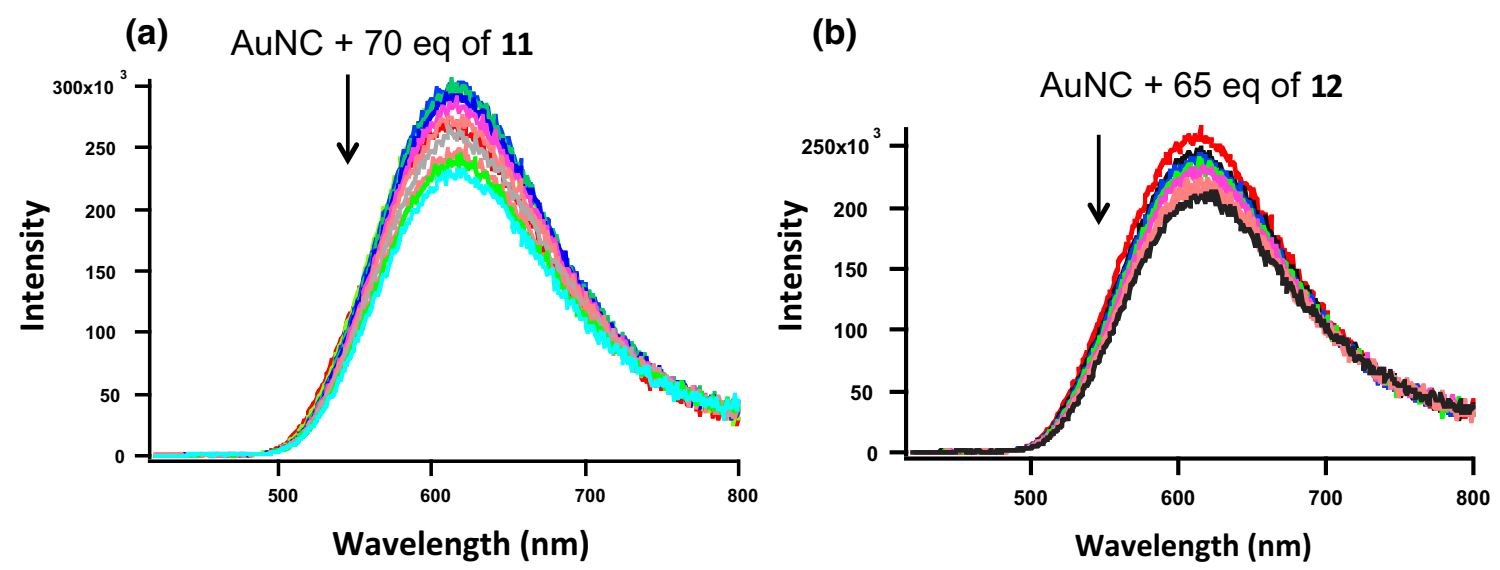

Figure 9. Fluorescence quenching of AuNC by N-methylpicolinium esters in methanol, using (a) 11 and (b) 12. Quenching in both cases were minimal.

and 12. Given that AuNC is able to reduce substrates having reduction potentials only above $-1.1 \mathrm{eV}$, the inability of the two esters $(-1.5 \mathrm{eV})$ to quench was not surprising. As expected, irradiation of a solution of AuNC and the two esters did not result in any reaction. We plan to pursue this study employing more easily reducible triggers such as methyl viologen, etc. and in the presence of an additional electron donor.

One other reaction that worked best with AuNP was the thermal isomerization of $c$ is- to trans-azobenzene. ${ }^{37}$ The process is illustrated in Scheme 4. cis-Azobenzene prepared photochemically from trans-azobenzene slowly reversed to the trans isomer in a week (Figure S15a in Supplementary Information). However, when it is included within an organic capsule (octa acid) it took over two months (Figure S16b, Supplementary Information). However, most interestingly, when the capsule containing cis-azobenzene was stirred with AuNP the isomerization occurred within an hour (Figure S16c, Supplementary Information). Clearly, the AuNP hastened the isomerization, presumably through an electron transfer process from AuNP to the ground state 


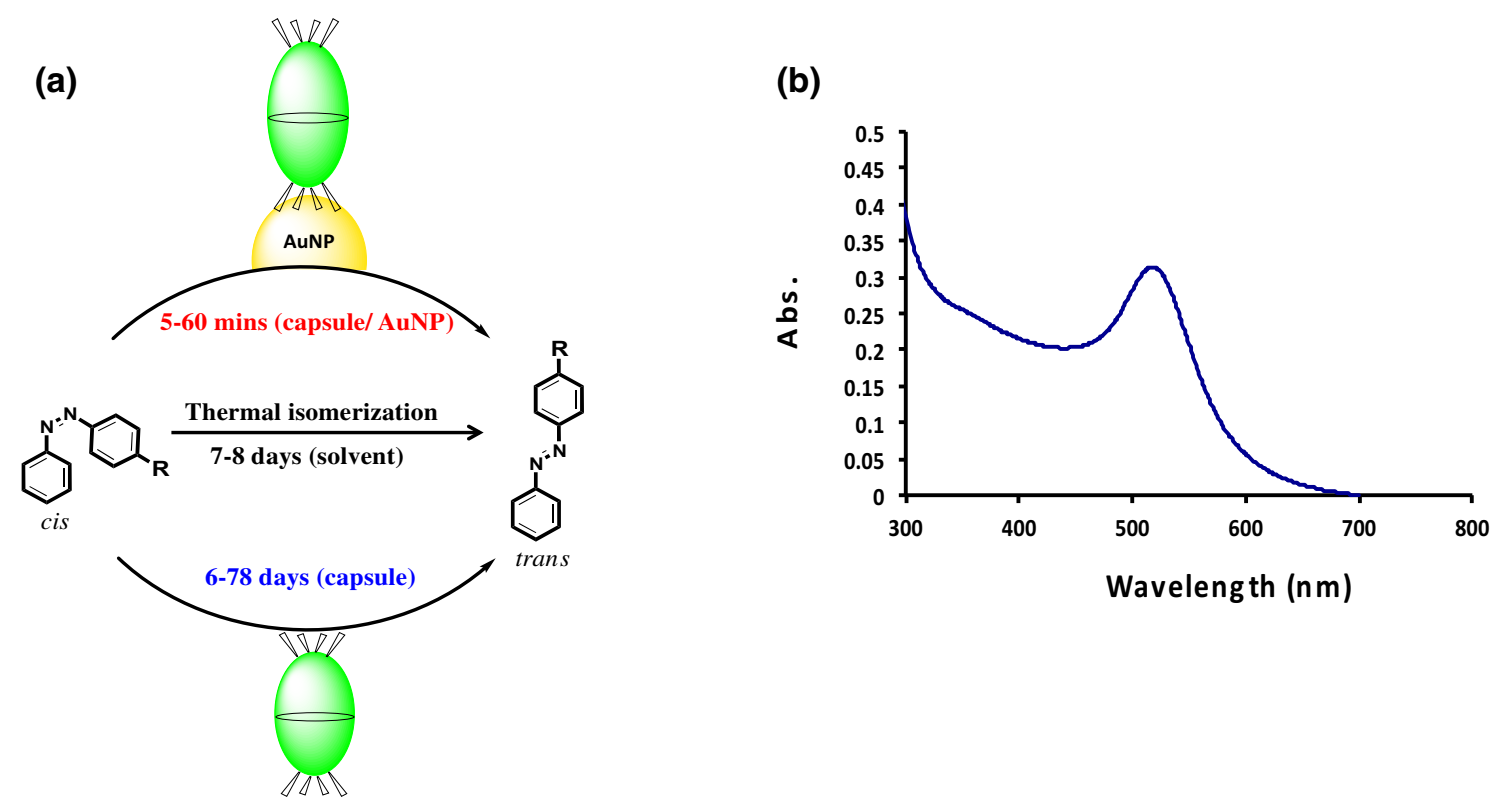

Scheme 4. (a) Environment-dependent isomerization of cis-azobenzene. (b) Absorption spectrum of AuNP used in the study.

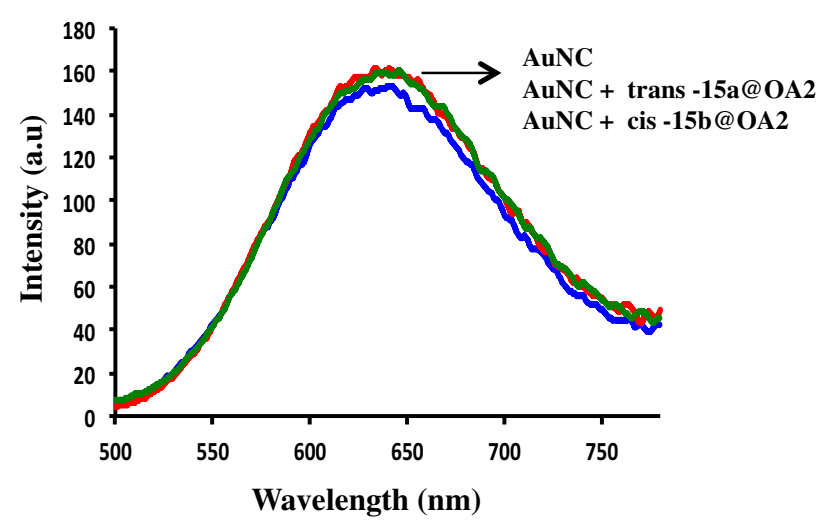

Figure 10. Emission spectra of AuNC solution after addition of both cis and trans isomer of $15 @ \mathrm{OA}_{2}$.

cis-azobenzene. We wished to probe whether AuNC will do the same. With this in mind, cis-azobenzene encapsulated octa acid capsule was stirred with AuNC in water for several hours. There was no conversion of cis to trans in presence of AuNC. From this result, it is clear that AuNC behaves distinctly differently from AuNP. To test whether excited AuNC will initiate isomerization by transferring an electron to octa acid encapsulated cis-azobenzene we performed AuNC emission quenching experiments. As shown in Figure 10, the addition of octa acid encapsulated cis-azobenzene or trans-azobenzene did not result in quenching of AuNC emission. Clearly, AuNC even from the excited state does not initiate the isomerization of azobenzenes. This is consistent with the results with several quenchers (Scheme 2) discussed above.
The reduction potential of azobenzenes $(\sim-1.4 \mathrm{eV})$ are too low for AuNC to reduce them and initiate isomerization.

\section{Conclusions}

Our recent interest in visible light photocatalysis prompted us to identify stable molecules/materials that would absorb in the visible spectrum and function as sensitizers and initiate transformations of organic molecules by transferring either energy and/or electron. ${ }^{38-41}$ This led us to search for stable and clean gold nanoparticles and nanoclusters. These well-explored materials have absorptions in the visible spectrum and act as electron and energy transfer agents. In spite of their dominance in materials science, to our knowledge, they have not been commonly used as sensitizers to initiate organic photoreactions. Thanks to the report by $\mathrm{Xie},{ }^{23,24}$ AuNC that absorbs in the visible region can be easily synthesized. AuNC thus synthesized consists of a mixture of clusters of different sizes. The above AuNC has limited ability to serve as an electron donor. It can reduce organic molecules whose reduction potentials are above $\sim-1.1 \mathrm{eV}$. Based on the fluorescence quenching experiments we believe that AuNC has only limited ability to serve as energy transfer sensitizer. It is quite likely that well-defined AuNC such as $\mathrm{Au}_{25} \mathrm{NC}$ will have better ability to reduce wide ranging substrates and would serve as energy transfer sensitizers. We plan to continue our search for the stable visible light absorbing 
sensitizers (catalysts) that would find use in the current burgeoning field of VLPC.

\section{Supplementary Information (SI)}

All experimental details that include synthesis and characterization of AuNC, procedures for recording emission and lifetime are provided. Supplementary Information is available at www.ias.ac.in/chemsci.

\section{Acknowledgements}

V.R. is thankful to the National Science Foundation (CHE1807729), USA for financial support.

\section{References}

1. Yoon T P 2016 Photochemical Stereocontrol Using Tandem Photoredox-Chiral Lewis Acid Catalysis Acc. Chem. Res. 492307

2. Ramamurthy V and Sivaguru J 2016 Supramolecular Photochemistry as a Synthetic Tool: Photocycloaddition Chem. Rev. 1169914

3. Prier C K, Rankic D A and MacMillan D W C 2013 Visible Light Photoredox Catalysis with Transition Metal Complexes: Applications in Organic Synthesis Chem. Rev. 1135322

4. Turro N J, Ramamurthy V and Scaiano J C 2010 Modern Molecular Photochemistry of Organic Molecules (Sausalito CA: University Science Books)

5. Katz E and Willner I 2004 Integrated NanoparticleBiomolecule Hybrid Systems: Synthesis Properties and Applications Angew. Chem. Int. Ed. 436042

6. Giljohann D A, Seferos D S, Daniel W L, Massich M D, Patel P C and Mirkin C A 2010 Gold Nanoparticles for Biology and Medicine Angew. Chem. Int Ed. 493280

7. Nguyen D T, Kim D-J and Kim K-S 2011 Controlled Synthesis and Biomolecular Probe Application of Gold Nanoparticles Micron 42207

8. Perez-Juste J, Pastoriza-Santos I, Liz-Marzan L M and Mulvaney P 2005 Gold Nanorods: Synthesis Characterization and Applications Coord. Chem. Rev. 2491870

9. Eustis S and EI-Sayed M A 2006 Why Gold Nanoparticles Are More Precious Than Pretty Gold: Noble Metal Surface Plasmon Resonance and Its Enhancement of the Radiative and Nonradiative Properties of Nanocrystals of Different Shapes Chem. Soc. Rev. 35209

10. Liz-Marzan L M 2006 Tailoring Surface Plasmons through the Morphology and Assembly of Metal Nanoparticles Langmuir 2232

11. Daniel M-C and Astruc D 2003 Gold Nanoparticles: Assembly Supramolecular Chemistry Quantum-SizeRelated Properties and Applications toward Biology Catalysis and Nanotechnology Chem. Rev. 104293

12. Zheng J, Nicovich P R and Dickson R M 2007 Highly Fluorescent Noble-Metal Quantum Dots Аnпи. Rev. Phys. Chem. 58409

13. Shang L, Dong S and Nienhaus G U 2011 Ultra-Small Fluorescent Metal Nanoclusters: Synthesis and Biological Applications Nano Today 6401
14. Jin R 2010 Quantum Sized Thiolate-Protected Gold Nanoclusters Nanoscale 2343

15. Templeton A C, Wuelfing W P and Murray R W 2000 Monolayer-Protected Cluster Molecules Acc. Chem. Res. 3327

16. Yau S H, Varnavski O and Goodson 2013 T An Ultrafast Look at $\mathrm{Au}$ Nanoclusters Acc. Chem. Res. 46 1506

17. Kohlmann O, Steinmetz W E, Mao X-A, Wuelfing W P, Templeton A C, Murray R W and Johnson C S 2001 Nmr Diffusion Relaxation and Spectroscopic Studies of Water Soluble Monolayer-Protected Gold Nanoclusters J. Phys. Chem. B 1058801

18. Parker J F, Fields-Zinna C A and Murray R W 2010 The Story of a Monodisperse Gold Nanoparticle: $\mathrm{Au}_{25} \mathrm{l}_{18}$ Acc. Chem. Res. 431289

19. Huang T and Murray R W 2001 Visible Luminiscence of Water-Soluble Monolayer-Protected Gold Clusters $J$. Phys. Chem. B 10512498

20. Yau S H, Varnavski O, Gilbertson J D, Chandler B, Ramakrishna G and Goodson T 2010 Ultrafast Optical Study of Small Gold Monolayer Protected Clusters: A Closer Look at Emission J. Phys. Chem. C 114 15979

21. Link S, Beeby A, FitzGerald S, EI-Sayed M A, Schaaff T G and Whetten R L 2002 Visible to Infrared Luminescence from a 28-Atom Gold Cluster J. Phys. Chem. $B 1063410$

22. Govindaraju S, Ankireddy S R, Viswanath B, Kim J and Yun K 2017 Fluorescent Gold Nanocluster for Selective Detection of Dopamine in Cerebrospinal Fluid Sci. Rep. 71

23. Luo Z, Yuan X, Yu Y, Zhang Q, Leong D T, Lee J Y and Xie J 2012 From Aggregation-Induced Emission of $\mathrm{Au}(\mathrm{I})$-Thiolate Complexes to Ultrabright $\mathrm{Au}(0) @ \mathrm{Au}(\mathrm{I})-$ Thiolate Core-Shell Nanoclusters J. Am. Chem. Soc. 134 16662

24. Yuan X, Zhang B, Luo Z, Yao Q, Leong D T, Yan N and Xie J 2014 Balancing the Rate of Cluster Growth and Etching for Gram-Scale Synthesis of Thiolate-Protected $\mathrm{Au}_{25}$ Nanoclusters with Atomic Precision Angew. Chem. Int. Ed. 531

25. Diaz S A, Hastman D A, Medintz I L and Oh E 2017 Understanding Energy Transfer with Luminescent Gold Nanoclusters: A Promising New Transduction Modality for Biorelated Applications J. Mater. Chem. B 2017 7907

26. Oh E, Huston A L, Shabaev A, Efros A, Currie M, Susumu K, Bussmann K, Goswami R, Fatemi F K and Medintz IL 2016 Energy Transfer Sensitization of Luminescent Gold Nanoclusters: More Than Just the Classical Forster Mechanism Sci. Rep. 61

27. Chen Y-S and Kamat P V 2014 Glutathione-Capped Gold Nanoclusters as Photosensitizers Visible Light-Induced Hydrogen Generation in Neutral Water J. Am. Chem. Soc. 1366075

28. Stamplecoskie K G, Chen Y-S and Kamat P V 2014 Excited-State Behavior of Luminescent GlutathioneProtected Gold Clusters J. Phys. Chem. C 118 1370

29. Chen Y-S, Choi H and Kamat P V 2013 Metal-ClusterSensitized Solar Cells A New Class of Thiolated Gold 
Sensitizers Delivering Efficiency Greater Than 2\% J. Am. Chem. Soc. 1358822

30. Hasobe T, Imahori H, Kamat P V, Ahn T K, Kim S K, Kim D, Fujimoto A, Hirakawa T and Fukuzumi S 2005 Photovoltaic Cells Using Composite Nanoclusters of Porphyrins and Fullerenes with Gold Nanoparticles $J$. Am. Chem. Soc. 1271216

31. Fan W and Leung M K H 2016 Recent Development of Plasmonic Resonance-Based Photocatalysis and Photovoltaics for Solar Utilization Molecules 21 180/1

32. Kawasaki H, Kumar S, Li G, Zeng C, Kauffman D R, Yoshimato J, Iwasaki Y and Jin R 2014 Generation of Singlet Oxygen by Photoexcited $\mathrm{Au}_{25}(\mathrm{Sr})_{18}$ Clusters Chem. Mater. 262777

33. Ipe B I, Thomas K G, Barazzouk S, Hotchandani S and Kamat P V 2002 Photoinduced Charge Separation in a Fluorophore-Gold Nanoassembly J. Phys. Chem. B 106 18

34. Thomas K G and Kamat P V 2000 Making Gold Nanoparticles Glow: Enhanced Emission from a Surface-Bound Fluoroprobe J. Am. Chem. Soc. 122 2655

35. Thomas K G and Kamat P V 2003 ChromophoreFunctionalized Gold Nanoparticles Acc. Chem. Res. 36 888
36. Borak J B, Lopez-Sola S and Falvey D E 2008 Photorelease of Carboxylic Acids Mediated by VisibleLight-Absorbing Gold-Nanoparticles Org. Lett. 10 457

37. Mohan Raj A and Ramamurthy V 2017 Volume Conserving Geometric Isomerization of Encapsulated Azobenzenes in Ground- and Excited States and as Radical Ion Org. Lett. 196116

38. Ueno K and Misawa H Surface 2013 Plasmon-Enhanced Photochemical Reactions J. Photochem. Photobiol. C 15 31

39. Scaiano J C and Stamplecoskie K 2013 Can Surface Plasmon Fields Provide a New Way to Photosensitize Organic Photoreactions? From Designer Nanoparticles to Custom Applications J. Phys. Chem. Lett. 4 1177

40. Sarina S, Waclawik E R and Zhu H 2013 Photocatalysis on Supported Gold and Silver Nanoparticles under Ultraviolet and Visible Light Irradiation Green Chem. 15 1814

41. Zhou N, López-Puente V, Wang Q, Polavarapu L, Pastoriza-Santos I and Xu Q-H 2015 Plasmon-Enhanced Light Harvesting: Applications in Enhanced Photocatalysis Photodynamic Therapy and Photovoltaics RSC Adv. 529076 